Special issue of the 2nd International Conference on Computational and Experimental Science and Engineering (ICCESEN 2015)

\title{
Performance Analysis of CoS of Bridged WorldFIP and ATM Networks for Real Time Communication
}

\author{
T. Aydogan ${ }^{a}, *$ I. OzCEliK ${ }^{b}$, I. ERTURK ${ }^{c}$ AND H. EKIZ ${ }^{d}$ \\ ${ }^{a}$ Suleyman Demirel University, Software Eng. Department, Isparta, Turkey \\ ${ }^{b}$ Sakarya University, Computer Eng. Department, Sakarya, Turkey \\ ${ }^{c}$ Kocaeli University, Electronic-Computer Edu. Department, Kocaeli, Turkey \\ ${ }^{d}$ Suleyman Sah University, Computer Eng. Department, Istanbul, Turkey
}

\begin{abstract}
The implementation of the remote control, monitoring and maintenance of industrial automation systems based on real-time criteria is becoming more difficult as the distance increases. ATM is an important transport, switching, network management and backbone technology that offers different solutions to real-time communication with different classes of service. In this study, the effects on real-time communication of AAL $3 / 4$ and AAL 5 classes of service of ATM were researched on a model in which the communication between WorldFIP industrial network nodes and ATM network nodes was provided over a bridge. First, a communication system consisting of a WorldFIP network, ATM network and bridge were modeled on a simulation tool and implemented. Afterwards, the system performance was analyzed under different loads within the classes of service AAL $3 / 4$ and AAL 5 . The analysis results have indicated that compared to AAL 5, AAL 3/4 better satisfies the real-time communication criteria. This result has shown that in both real-time access to a remote industrial automation systems and communication among remote industrial automation systems, ATM is preferable as a backbone and AAL $3 / 4$ as the class of service.
\end{abstract}

DOI: 10.12693/APhysPolA.130.412

PACS/topics: 01.20.+X, 07.05.TP

\section{Introduction}

Fieldbuses are communication networks intended for industrial use that connect field devices such as sensors and actuators and field controllers such as PLCs (programmable logic controllers), regulators, drive controllers, etc. and human-machine interfaces to each other [1]. There is a variety of fieldbuses such as ASI, BITBUS, CAN, DeviceNet, Hart, IEC-Fieldbus, InterBus-S, LonWorks, P-NET, PROFIBUS, Rackbus, SERCOS, SDS, TRON and WorldFIP [2]. All of the fieldbuses implement communication in accordance with real-time communication criteria, which is one of the requirements of industrial automation systems. Message latency time, one of the important parameters of real-time communication, is $100-300 \mathrm{~ms}$ in process technologies, 10-100 $\mathrm{ms}$ in manufacturing technologies and 1-10 $\mathrm{ms}$ in drive systems [3]. Message response time, another important parameter, is $10 \mathrm{~ms}$ in $34 \%, 1 \mathrm{~ms}$ in $30 \%, 100 \mathrm{~ms}$ in $14 \%$, higher than $100 \mathrm{~ms}$ in $12 \%$ and $0.1 \mathrm{~ms}$ in $10 \%$ of fieldbuses [4].

WorldFIP (world factory instrumentation protocol) is a fieldbus used in industrial manufacturing, power, process, transportation and other fields. Defined as an international communication standard, WorldFIP offers real-time data communication using three-layer fieldbus architecture. The physical layer of WorldFIP has S1 $(31.25 \mathrm{Kbit} / \mathrm{s}), \mathrm{S} 2(1 \mathrm{Mbit} / \mathrm{s})$ and S3 $(2.5 \mathrm{Mbit} / \mathrm{s})$ data transmissions on shielded twisted-pair and $5 \mathrm{Mbit} / \mathrm{s}$ on

\footnotetext{
* corresponding author; e-mail: tuncayaydogan@sdu.edu.tr
}

fiber optic. The data link layer provides variable exchange and message transfer services. The application layer implements messaging and message control services. A WorldFIP network consists of a bus arbitrator (BA), station nodes with producer $(\mathrm{P}) /$ consumer $(\mathrm{C})$ features and a serial bus $[5,6]$.

Asynchronous transfer mode (ATM) is a backbone communication technology that ensures the communication of different types of user services such as data, sound and image using different classes of service (CoS). ATM has three-layer architecture that possesses the functions of the first two layers of the OSI (open system interconnection) model. The physical layer consists of physical medium and transmission convergence sub-layers. An ATM layer ensures general flow control, generation and extraction of a cell header, multiplexing and demultiplexing of cell virtual path/channel identifier. An ATM adaptation layer (AAL) consists of layers of segmentation and reassembly and convergence sublayer and is responsible for the adaptation of the data in upper layers to ATM cells. In AAL, different $\mathrm{CoS}$ defined as AAL 1, AAL 2, AAL $3 / 4$ and AAL 5 have been created depending on the timing relationship between the cell's source and destination, bit transfer rate and connection mode. In ATM, data is transported in packets referred to as cells that have a 53 byte fixed length, using packet switching technology [7].

Modern industrial automation systems develop in the direction of merging local industrial automation systems located at a geographical distance to one another and equipped with the same or different technologies and their conversion into distributed automation systems. 
These systems require real-time remote connection to local automation systems for remote control, configuration, monitoring and maintenance of distributed automation systems [8]. When the distance between the networks increases, it is difficult to maintain real-time communications.

In the literature there are solutions involving Ethernet [9], FDDI (fiber distributed data interface) [10] and ATM [11-14] communication technologies for connections among remote fieldbuses. Moreover, ATM is indicated to be one of the backbone technologies suitable for WorldFIP [15] and a study is available, in which WorldFIP was controlled and monitored over ATM using PC gateway [16].

Protocol converting devices are used to establish connections between networks systems with different technologies. Bridges are used to connect local area networks (LANs) with the same or different technologies and to generate extended LANs. Because fieldbuses have the first three layers of an OSI, they can be connected to another network by a bridge [1, 17-19].

The purpose of this study was to identify the ATM class of service that could provide real-time communication between remote WorldFIP fieldbus nodes and ATM network nodes. A communication system was designed for the study that consisted of WorldFIP and ATM networks and a WorldFIP/ATM bridge. The designed system was modeled using a NetworkII.5 simulation tool and separately simulated so that it could transmit a traffic model generated under various loads at CoS AAL 3/4 and AAL 5. The simulation results were analyzed and the extent of satisfaction by the CoS AAL $3 / 4$ and AAL 5 and industrial automation real-time communication criteria was evaluated.

\section{The WorldFIP/ATM communication model}

Figure 1 illustrates the communication model designed for the study. The model consists of an automation system created using the WorldFIP fieldbus, an ATM network capable of performing functions such as remote monitoring, maintenance and control of the automation system and a WorldFIP/ATM bridge capable of ensuring communication between the two networks with different technologies.

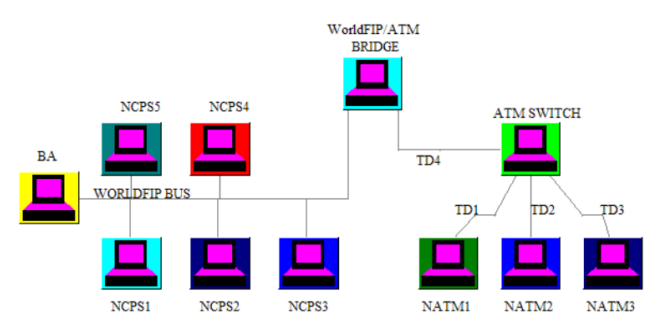

Fig. 1. WorldFIP/ATM networking model.

WorldFIP network model in Fig. 1 consists of five NCPSn nodes and one BA that communicate with the WorldFIP fieldbus. Each NCPSn node with $\mathrm{C} / \mathrm{P}$ features has been modeled using an actuator and sensor connection. The BA and all nodes were modeled using algorithms that could manage such different traffic loads $[6,20]$. In the model, the WorldFIP bus was configured according to the S3 data transmission rate while the nodes were configured in accordance with $80 \mathrm{MHz}$ WorldFIP chip features.

The ATM network model in Fig. 1 consists of three NATMn personal computer nodes and one switch. The ATM network has been separately modeled for the CoS AAL $3 / 4$ and AAL 5. In the model, the buses TD1, TD2 and TD3 were configured at the data transmission rate of $25 \mathrm{Mbps}$, bus TD4 at $155 \mathrm{Mbps}$ and switch IBM 8285 was modeled in accordance with the NwaysWorkgroup switch features [21].

Because WorldFIP and ATM protocol features are dissimilar, the WorldFIP/ATM bridge model in Fig. 1 was designed so as to possess all the features related to the functions of a two-port transparent-translating local bridge [17, 19, 22].

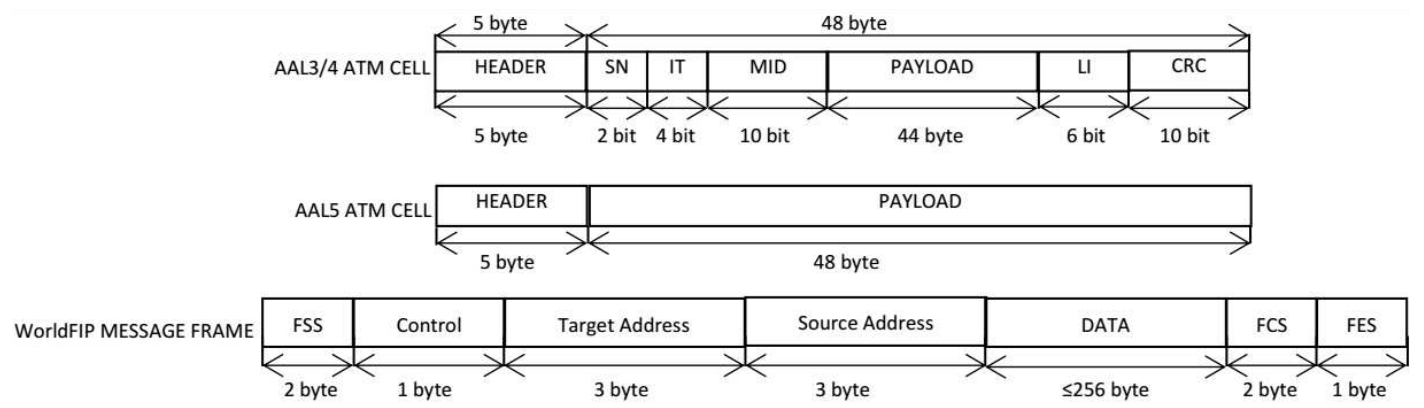

Fig. 2. Formats of AAL 3/4, AAL 5 ATM cells and WorldFIP frames.

Figure 2 indicates that the WorldFIP frame format is different from the formats of ATM cells of CoS AAL 3/4 and AAL 5. Finally, in the modeling of bridge entities and algorithms, the conversion from WorldFIP frame format into AAL 3/4 and AAL 5 ATM cell formats and from the two ATM cell formats into WorldFIP frame format was conducted using three-step processes by converting as follows. 
Step 1: Discarding fields that are unnecessary for other format.

Step 2: Reconstructing fields that are necessary for other format.

Step 3: Producing new fields to other format.

\section{Simulation and performance analysis of the WorldFIP/ATM networking model for ALL 3/5 and AAL 5 data traffics}

The model of the designed system, shown in Fig. 1, and its simulation were implemented using NetworkII.5 software, programmed using Simscript II [23, 24]. The used software has features that make it capable of modeling the lower layers of OSI and real-time systems using the discrete event simulation technique [11, 14]. These features are sufficient for WorldFIP, ATM network and bridge modelling [22].

Local $(\mathrm{L})$ and remote $(\mathrm{R})$ message traffics have been modeled on both networks in order to evaluate system performance. Traffic among the networks' own nodes was defined as "local messages" while their traffic with the nodes of another network was defined as "remote messages".

Network message traffic features were defined, modeled and simulated at the rates of $70 \%-30 \%, 50 \%-$ $50 \%$ and $30 \%-70 \%$ locally/remotely for WorldFIP, and at the rates of $70 \%-30 \%, 50 \%-50 \%$ and $30 \%-70 \%$ locally/remotely for ATM. Also they were defined by changing the mean value of exponential distribution so as to generate values with variable data fields up to 256 bytes for WorldFIP, and 53 bytes fixed length for ATM.

Analyses were conducted in accordance with bus utilization, process time and cycle time of both WorldFIP and ATM under different local/remote message loads.

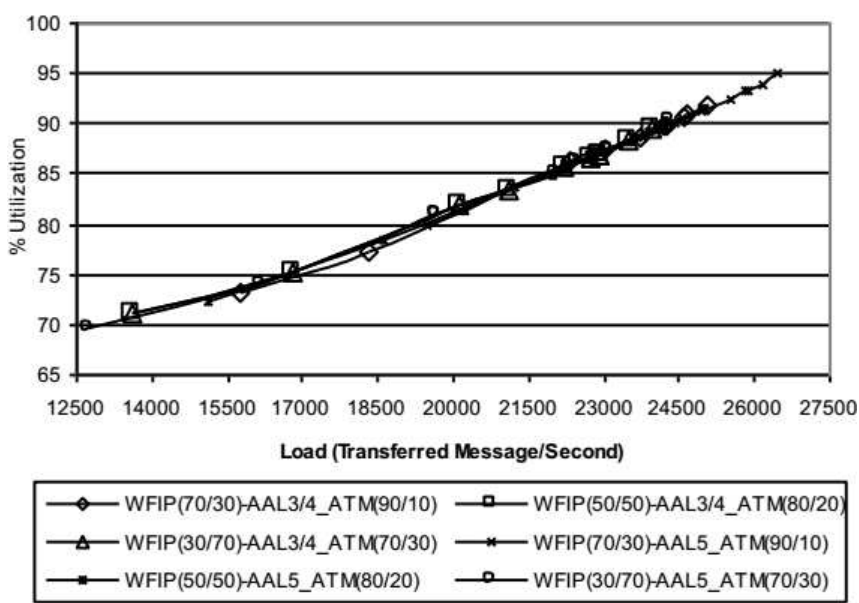

Fig. 3. WorldFIP bus utilization rates.

Figure 3 illustrates the utilization rates of WorldFIP bus with uses of ATM cell formats at CoS AAL $3 / 4$ and AAL 5 under different loads. Bus utilization was in the range of $71 \%-92 \%$ with AAL $3 / 4$ and $69 \%-95 \%$ with AAL 5. In the WorldFIP network traffic, messaging is conducted using six different frames of different sizes from 6 to 268 bytes. The fact that utilization rates of the service class AAL 5 are slightly higher than those of the service class AAL $3 / 4$ is caused by shorter idle time on the line as a result of the higher data load quantity used in the AAL 5 cell.

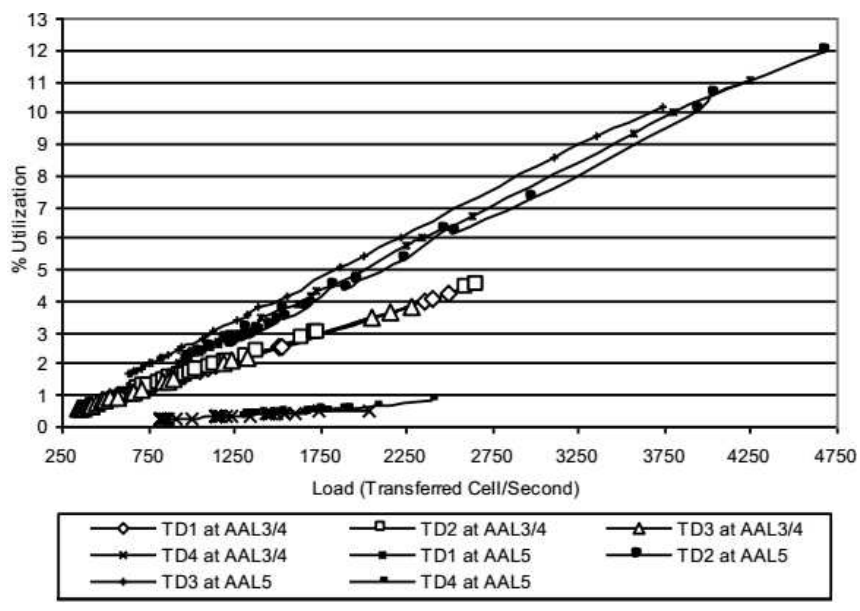

Fig. 4. Utilization rates of TDn ATM buses.

Figure 4 illustrates utilization rates of ATM buses with labels TD1, TD2, TD3 and TD4 with the use of ATM cell formats of CoS AAL $3 / 4$ and AAL 5 under different loads. TD1, TD2 and TD3 utilization rates varied in the range from $0.5 \%$ to $4.5 \%$ with AAL $3 / 4$ and from $1.7 \%$ to $12 \%$ with AAL 5 . TD4 utilization rates varied in the range from $0.2 \%$ to $0.5 \%$ with $\mathrm{AAL} 3 / 4$ and from $0.3 \%$ to $0.8 \%$ with AAL 5 . The reason why these values are generally lower than those of WorldFIP bus is the high data transmission rates of ATM buses and small cell size. The reason why the utilization rates of the service class AAL 5 are slightly higher than those of AAL $3 / 4$ is the longer utilization time of the bus as a result of higher data load quantity of the AAL 5 cell.

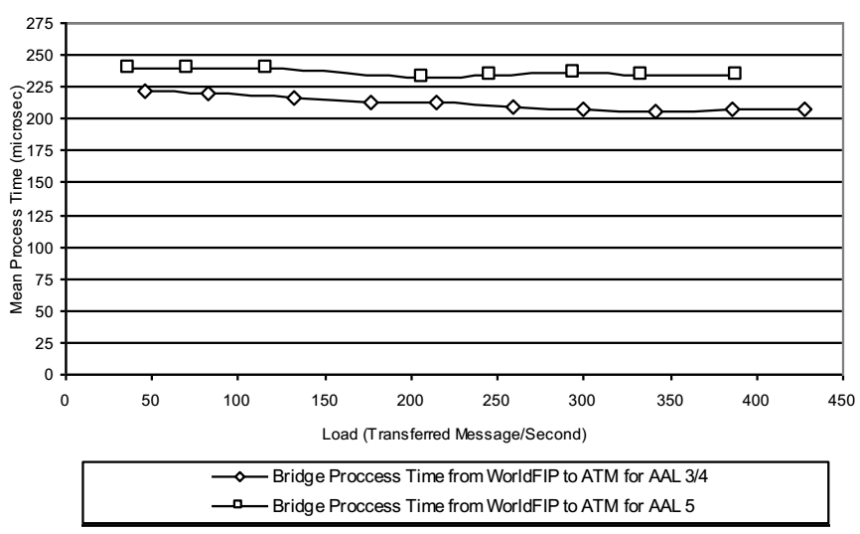

Fig. 5. Bridge process time from WorldFIP to ATM.

Figure 5 illustrates the process time from WorldFIP to ATM. Process time is the time that passes from the 
receipt of the message at one port of the bridge. Process time has ranged from 207 to $221 \mu$ s with the use of AAL $3 / 4$ and from 234 to $238 \mu$ s with the use of AAL5. The reason for a slightly longer process time for service class AAL 5 compared to that of the service class AAL $3 / 4$ is the larger size of data kept waiting at the bridge buffers due to the higher data load of the AAL 5 cell format, i.e. the increase in the waiting time in the buffer and data transmission times.

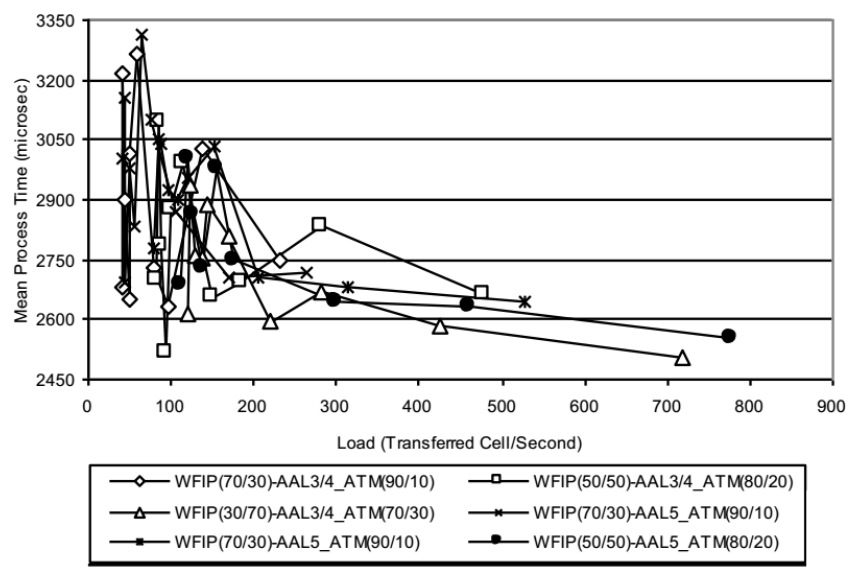

Fig. 6. Bridge process time from ATM to WorldFIP.

Figure 6 illustrates the process time from ATM to WorldFIP. The process time has ranged from 2.5 to $2.7 \mathrm{~ms}$ with the use of AAL $3 / 4$ and from 2.5 to $3 \mathrm{~ms}$ with the use of AAL 5 . It can been seen from the graph that as the ratio of remote messages increases, the number of messages processed on the bridge also increases. The uneven variation on the graph and higher process times than those illustrated in Fig. 5 are attributable to the waits in the WorldFIP side buffer of the bridge. Slightly higher values for AAL 5 compared to those for AAL $3 / 4$ are attributable to larger data kept waiting in the buffers due to the greater data load of the AAL 5 cell format and to the longer duration of the process of conversion into WorldFIP, i.e. increase in the times of waiting in the buffer and data transmission.

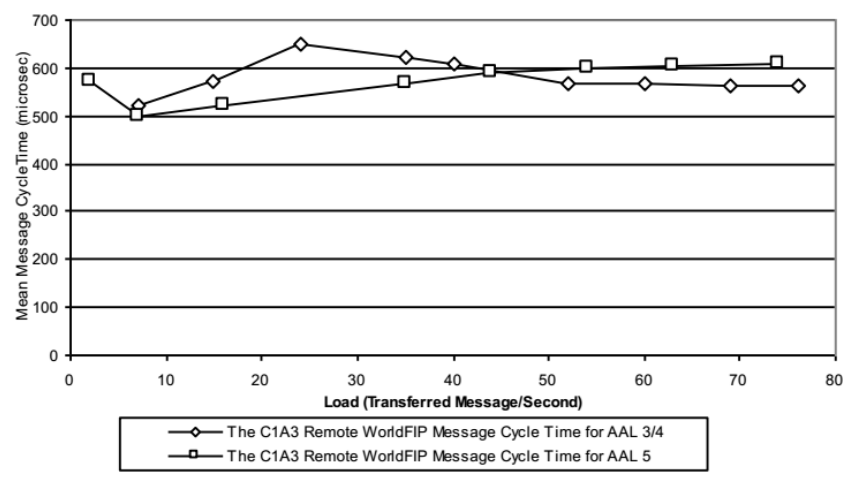

Fig. 7. Cycle time of the remote WorldFIP message.
Figure 7 illustrates the cycle time from WorldFIP to ATM of a remote WorldFIP message labeled as C1A3 from NCPS1 to NATM3. The cycle time ranged from 523 to $650 \mu \mathrm{s}$ with AAL $3 / 4$ and from 499 to $607 \mu \mathrm{s}$ with AAL 5. Since data is carried using less cells with AAL 5 compared to AAL 3/4, the total time required for a WorldFIP message to get to the target node was shorter with AAL 5 cells. This is more clearly observed in the graphs where the number of messages is low.

Figure 8 illustrates the cycle time from ATM to WorldFIP of a remote ATM message labeled as A3C5 from NATM3 to NCPS5. The cycle time ranged from 262 to $271 \mu$ s with AAL $3 / 4$ and from 337 to $345 \mu$ s with AAL 5. A different format and WorldFIP message frame conversion algorithm of an AAL 5 cell compared to those of an AAL $3 / 4$ cell are responsible for the delay experienced with AAL 5.

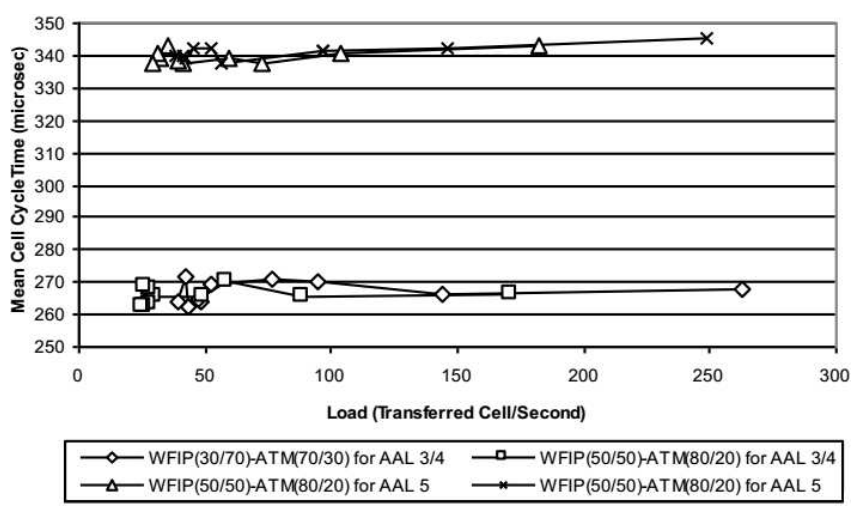

Fig. 8. Cycle time of the remote ATM message.

\section{Conclusions}

In this study, nodes on an ATM network were connected to a remote WorldFIP fieldbus automation system via a bridge and a model was designed allowing them to communicate using AAL $3 / 4$ and AAL 5 CoS features. Performance of the model was analyzed using discrete event simulation technique.

The reason for the difference between the analyses based on AAL 5 and AAL $3 / 4$ is the difference between cell formats and communication algorithms. Those differences affect the quantity of data kept waiting in buffers, conversion process time for WorldFIP/ATM and ATM/WorldFIP, buffer waiting time and data transmission times.

Results of the study have shown that ATM technology and AAL $3 / 4$ and AAL 5 CoS support the 1-10 ms message latency time [3] and that $64 \%$ of fieldbuses support the message response time obtained as 1-10 ms [4]. It has been further determined that as a CoS, AAL $3 / 4$ yields higher performance than AAL 5 and that WorldFIP / ATM bridge fieldbuses satisfy the real-time communication requirements, i.e. it is possible to remotely connect ATM networks to WorldFIP fieldbuses via bridges in order to monitor, maintain and control them. 


\section{References}

[1] J.P. Thomesse, in: 2005 IEEE Conference on Emerging Technologies and Factory Automation, IEEE, 2005, p. 1073.

[2] B. Galloway, G.P. Hancke, in: IEEE Communications Surveys \& Tutorials, Vol. 15, IEEE, 2013, p. 860.

[3] K. Etschberger, Controller Area Network, IXXAT Pres, Weingarten, Germany 2001.

[4] W. Lawrenz, CAN System Engineering from Theory to Practical Applications, Springer, 1997.

[5] A.V. Scott, W.J. Buchanan, in: Engineering of Computer Based Systems, 2000. (ECBS 2000) Proceedings, IEEE, Edinburgh 2000, p. 165.

[6] E. Tovar, F. Vasques, Comput. Ind. 44, 11 (2001).

[7] R. Handel, S. Schroder, M. Huber, ATM Networks, Concepts Protocols Applications, 3rd ed., AaddisonWesley, 1999.

[8] P. Neumann, Control Engineering Practice 15, 1332 (2007).

[9] F. Arjmandi, B. Moshiri, in: 2007 5th IEEE International Conference on Industrial Informatics, Vol. 1, IEEE, Vienna 2007, p. 213

[10] S. Cavalieri, A. Di Stefano, O. Mirabella, in: Local Computer Networks Proceedings, IEEE, Minneapolis 1994, p. 59.

[11] I. Özçelik, H. Ekiz, Comp. Stand. Interf. 30, 177 (2008).

[12] P. Zand, S. Chatterjea, J. Ketema, P. Havinga, in: Proc. 2012 IEEE 17th International Conference on Emerging Technologies \& Factory Automation (ETFA 2012), IEEE, Krakow 2012, p. 1.
[13] O. Kunert, in: Broadband Communications Proc., IEEE, Zurich 2000, p. 147.

[14] I. Özçelik, H. Ekiz, J. Netw. Comput. Appl. 30, 800 (2007).

[15] T. Wijnands, W. Herr, P. Ribeiro, in: ICALEPCS'99, Eds. D. Bulfone, A. Daneels, Trieste 1999, p. 176.

[16] R. Lauckner, R. Rausch, P. Ribeiro, T. Wijnands, in: Real Time Conference, IEEE NPSS, Sante Fe 1999, p. 194.

[17] M. Zitterbart, A.N. Tantawy, D.N. Serpanos, in Networking, IEEE/ACM Transactions on, Vol. 2, IEEE, 1994, pp. 352.

[18] F. Hallsall, Data Communication, Computer Networks, Open Systems, 4th ed., Addison-Wesley GM, USA 1996

[19] IEEE, IEEE Standard for Local and Metropolitan Area Networks Media Access Control (MAC) Bridges, IEEE, 2004, p. 1.

[20] P. Marino, M.A. Dominguez, F. Poza, J.B. Nogueira, in: EUROMICRO Conference Proc., Vol. 2, 1999, p. 334 .

[21] 8285 Nways ATM Workgroup Switch Support, www. networking.ibm.com/support/products.nsf/ techsupport/(8285)?OpenDocument.

[22] T. Aydogan, I. Ozcelik, A. Kutlu, H. Ekiz, Turk. J. Elec. Eng. Comp. Sci. 20, 425 (2012).

[23] W.J. Garrison, in: Simulation Conference Proceedings, Winter, 1990, p. 132.

[24] CACI, Network II.5 User's Manual, CACI Products Company, Release 12, 1997. 